\title{
Socio-demographic and epidemiological consideration of Africa's COVID-19 response: what is the possible pandemic course?
}

\author{
Africa is said to be the next possible hotspot for COVID-19. However, we believe this is still preventable. \\ Bamba Gaye, Stéphanie Khoury, Crystal W. Cene, Samuel Kingue, Roland N'Guetta, Camille Lassale, \\ Dadhi Baldé, Ibrahima Bara Diop, Jennifer Beam Dowd, Melinda C. Mills and Xavier Jouven
}

M any predicted a heavy toll of the COVID-19 pandemic on Africa. Its weakened health systems were harbingers of a terrible outcome. However, local expertise gathered from previous outbreaks and centralized public-health infrastructure with a clear action plan, in addition to a favorable demographic structure and climate, have put many countries in the continent in good standing to face this epidemic head on.

Accordingly, thus far, Africa seems to be affected only moderately by COVID- 19 . As of 3 June 2020, the virus had spread to all 54 countries in Africa, totaling to about 156,000 confirmed cases and 4,931 estimated deaths. The first case was reported in Egypt (on 14 February 2020), followed by Nigeria, which started the sporadic spread of the virus throughout the continent.

We have compiled information from World Health Organization data (Fig. 1) to provide an overview of expected numbers of deaths from COVID-19 in the 18 countries of the African Research Network, in addition to Morocco, Algeria, Egypt, Nigeria, Kenya and South Africa, compared with those in France, Italy, Germany, the USA, Singapore and South Korea.

Egypt, Algeria, Morocco and South Africa were predicted to have the most immediate risk of circulating the causative coronavirus (SARS-CoV-2) due to their having the greatest volume of imports and travel from China to their main cities. Accordingly, they now have some of the largest numbers of confirmed cases and deaths throughout Africa. South Africa has been hit the hardest by the pandemic, with a total (as of 3 June 2020) of 35,812 confirmed cases and 755 deaths, followed by Egypt, with 27,536 confirmed cases and 1,052 deaths, Morocco, with 7,910 confirmed cases and 206 deaths, and Algeria, with 9,626 cases and 667 deaths.

Most countries seem to reach a critical inflection point, after which cases and deaths grow exponentially (Fig. 2). In Italy, for example, on 20 February, confirmed cases of COVID-19 jumped from 11 to 132 . Most African countries we report on here seem to have not reached this inflection point, apart from Algeria and Egypt, which seem to have reached this inflection point on 13 March and since then have faced a new trajectory with an exponential spread of cases.

As predicted, Nigeria, Ethiopia, Sudan, Angola, Tanzania, Ghana and Kenya have not reported many cases yet, due to the moderate volume of travel from China to these countries. However, because they are highly vulnerability due to their lack of healthcare infrastructure, it is important for these countries to maintain close surveillance ${ }^{1}$. This is particularly true for Nigeria, Sudan and Chad: although their number of cases and deaths remains relatively low, it doubled in a very short period of time between the end of April to early May.

Finally, it is important to closely follow the countries in Africa with a low number of confirmed cases, such as Malawi, Zimbabwe, Angola, Namibia, Mauritania, etc., to better control the trajectory of this pandemic. Although these countries have not reported many cases so far, they are highly unlikely to be able to respond effectively if COVID-19 were to reach their country, due to weakened infrastructure.

\section{Effective lockdown measures}

One reason several African countries have fared better other parts of the world is their rapid implementation of strict lockdown measures. As of 23 April 2020, many African countries had taken early measures to control the spread of the disease, learning from the slow implementation of such measures in other affected areas and from their lived experience with outbreak preparedness.

Countries that have experienced the Ebola epidemic were also at an advantage in combating COVID-19. According to the World Health Organization's African Region Office, when the first COVID-19 case in sub-Saharan Africa was diagnosed in Nigeria, healthcare workers were ready to use contact-tracing strategies to halt the spread before it was too late. As soon as the case was confirmed, other countries around sub-Saharan Africa began to take dire measures, such as closing borders and minimizing public gatherings.

For example, Senegal took severe measures, such as closing its borders and ensuring large gatherings were minimized, at a very early stage, within days of the first reported case. Senegal was able to treat $60 \%$ (211 of 361) of their confirmed cases on 18 April, and by early June, they had only 45 reported deaths. Unlike that rapid response, Europe and the USA failed to act until the virus had spread to hundreds of people. Senegal is tightening restrictions to try to contain the outbreak.

As elsewhere in the world, questions have been raised about possible under-reporting of cases. By testing only patients who present with severe symptoms, and not testing patients at an early stage, many countries, including the USA, Italy, Spain, UK and France, have failed to identify and contain a considerable number of potential transmitters and, therefore, have increased the risk of transmission through asymptomatic people ${ }^{2}$.

Actions were taken by African government healthcare workforces to increase the number of tests per day, which led to the delivery of about 1 million tests to Africa as of 16 April 2020. Consequently, and of note, there has been an increase of $43 \%$ in the number of infections with SARS-CoV-2 detected by the PCR test.

More testing capability will probably be required ${ }^{3}$. Morocco, for example, is performing more than 1,000 tests per day and acquired 100,000 kits to increase the number of tests per day. Kenya is performing 


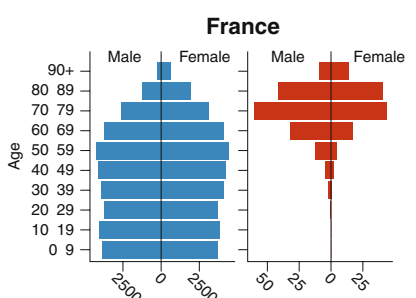

Singapore

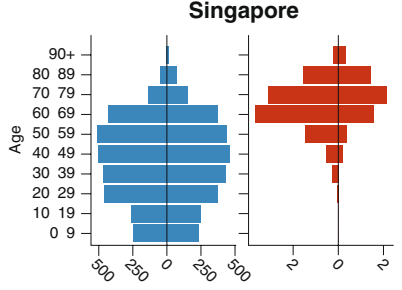

Kenya

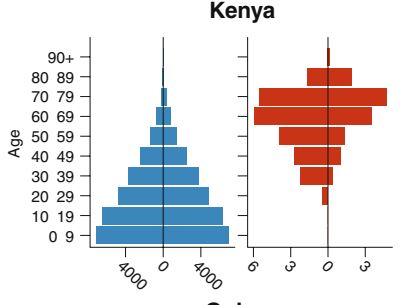

Guinea

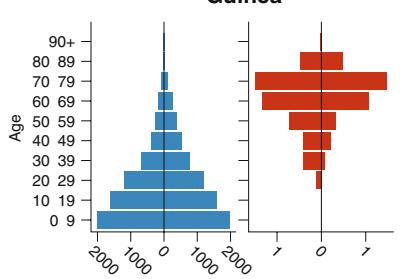

Cameroon

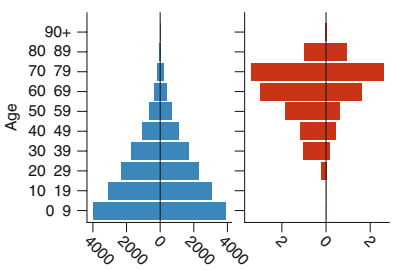

Rwanda

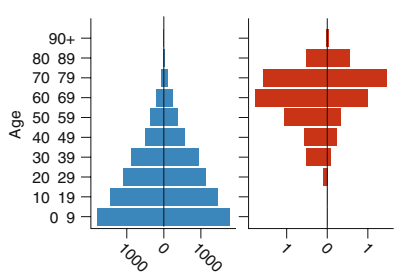

Italy

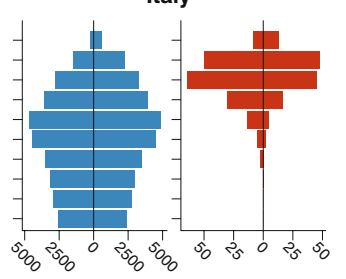

Algeria

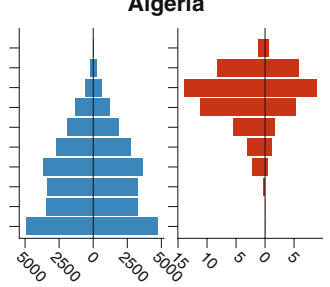

South Africa

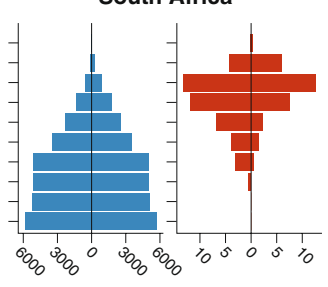

Burkina Faso

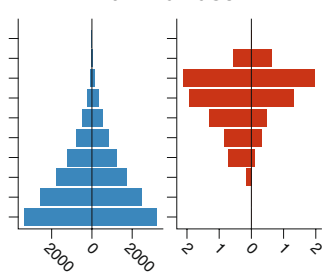

Chad

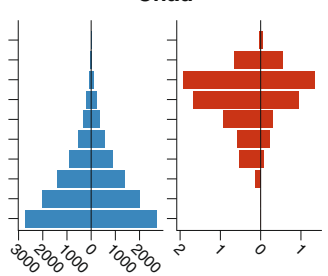

Sudan

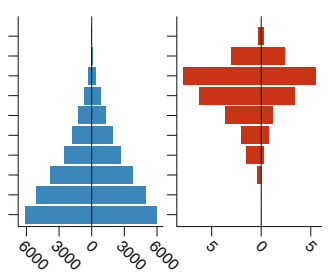

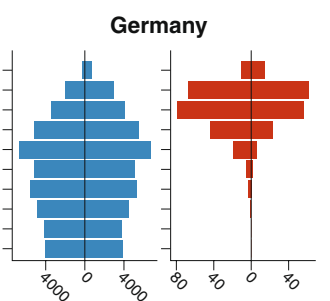

Morocco

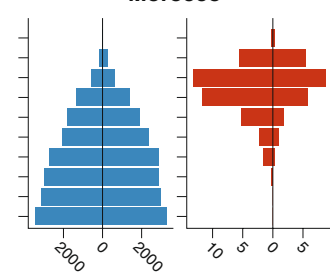

Mauritania

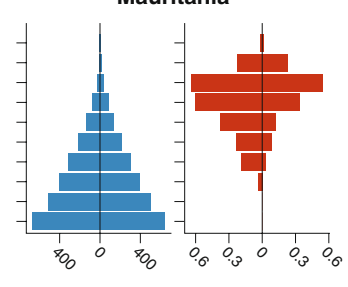

Mali

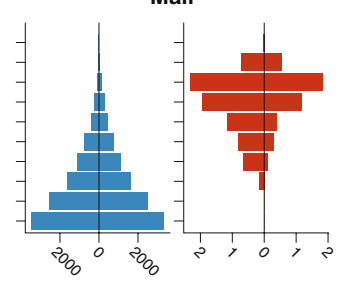

Democratic Republic

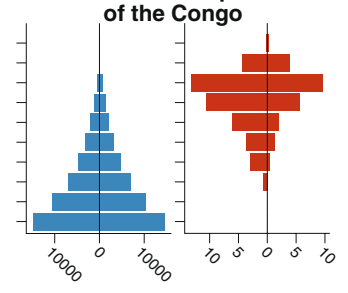

Gabon

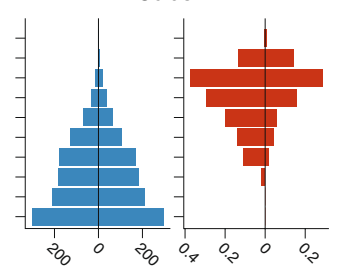

United States of America

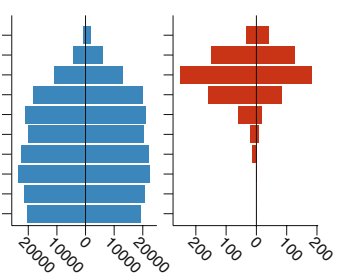

Egypt

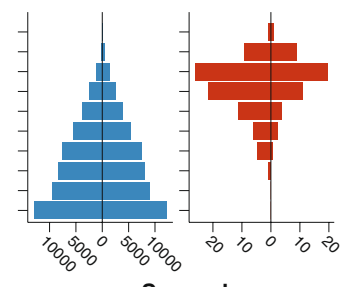

Senegal
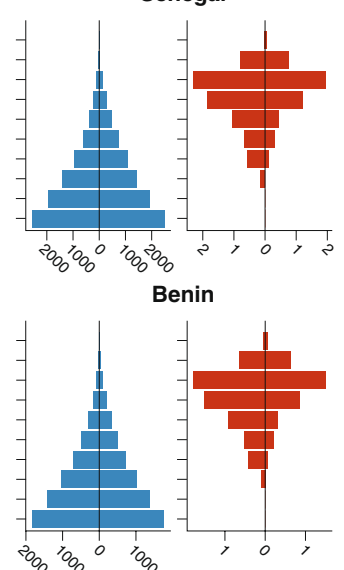

Ethiopia

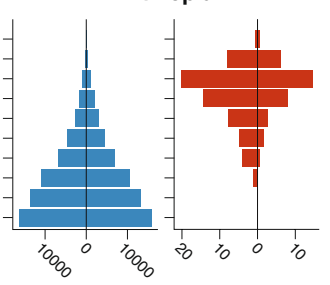

Population in thousands

Expected deaths in thousands
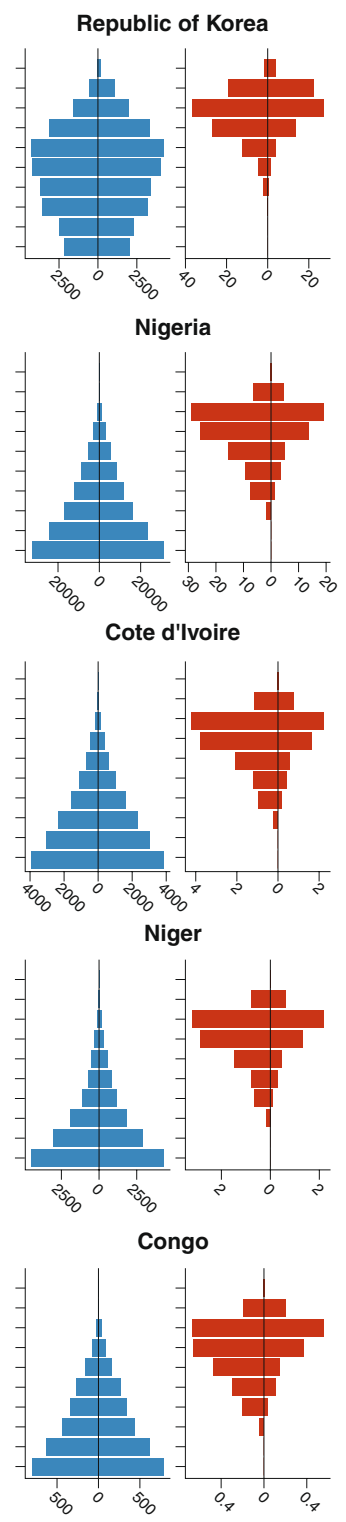

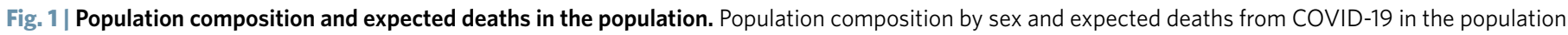
for 16 countries of the African Research Network, and Morocco, Algeria, Egypt, Nigeria, Kenya and South Africa, as well as France, Italy, Germany, the USA,

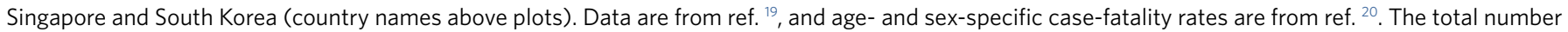

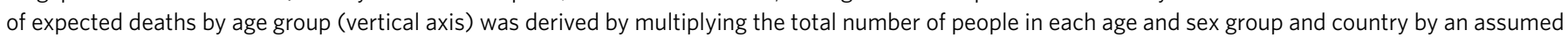
population infection rate of $10 \%$.

35,000 tests per day. Moreover, cases can go undetected in rural remote villages, where less testing has occurred. This has strong implications for the type of outreach and case-finding and containment approaches that should occur in rural remote villages.
Ensuring these countries have efficient surveillance and testing services can reduce the country-wide spread and, in turn, the transmission to other neighboring countries. This can be accomplished via checkpoints at the borders of each country for checking body temperature (even if a lot of spread is in the pre-symptomatic phase, or in asymptomatic people, and even though fever is not necessarily present in symptomatic people), and completion of a questionnaire by travelers, in addition to strategies to 
a

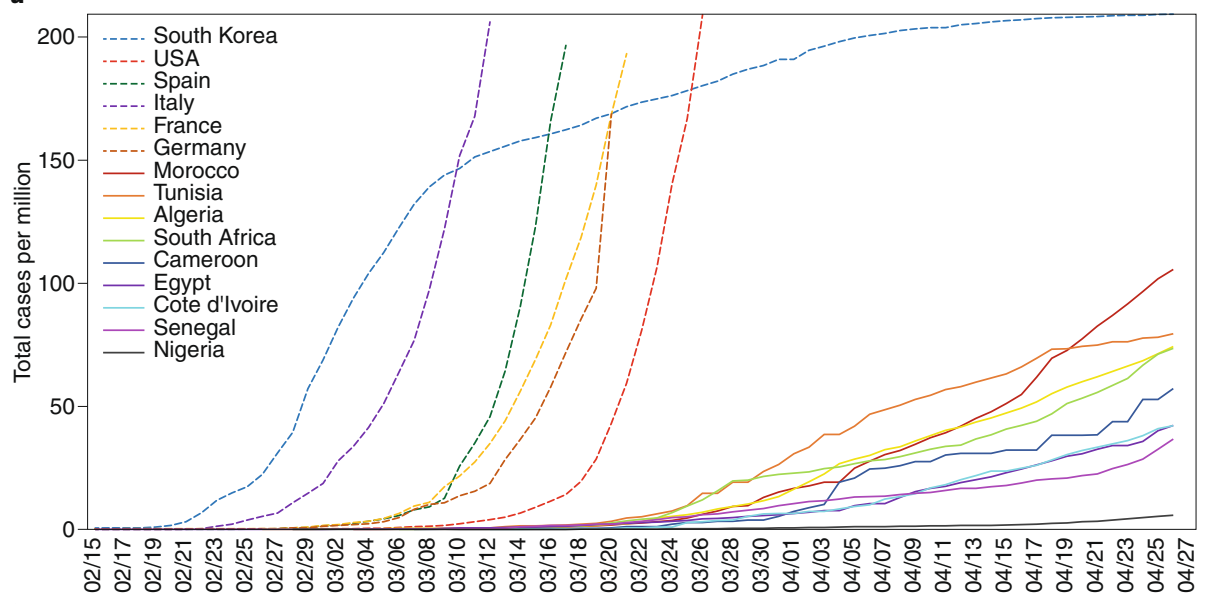

b

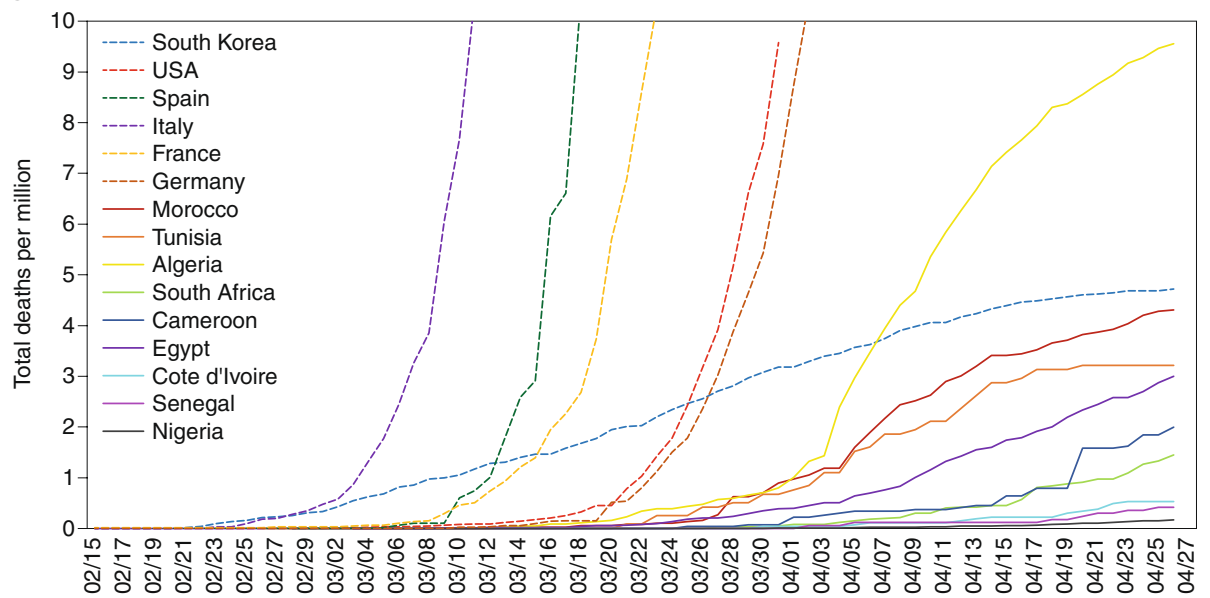

Fig. 2 | Confirmed cases and total death from COVID-19. Total COVID-19 cases (a) and total deaths from COVID-19 (b) over time (dates along horizontal axis: day/month) in Africa versus the USA, Europe and Asia (countries in key), adjusted for population size. Data analysis used data from the World Health Organization as of 26 April 2020. The dates start on 15 February 2020, the date of the first case in Africa (in Egypt). The six selected non-African countries correspond to countries with at least five cases on 14 March 2020. Data were analyzed with R software (version 3.6.3) using the packages ggplot2.

be implanted within each country (the Oxford tracker shows measures taken by governments, by country and date ${ }^{4}$ ).

The low number of severe cases of COVID-19 reported in Africa could also be due to its a younger demographic age structure. According to the United Nations, only $3 \%$ of the population in sub-Saharan Africa is over the age of 65 years, compared with $23 \%$ in Italy, for example. Given the strong link observed between older age and COVID-19 mortality, population age structure may be an important determinant of the prevalence and fatality rates of COVID-19 observed in Africa ${ }^{5}$ (Fig. 2).

Additionally, a plausible theory about the lower morbidity rate of COVID-19 in countries in Africa, as well as in Thailand and Vietnam, is that climate might have a role in the virulence of this disease. Temperature and humidity are known factors in the transmission of other coronaviruses (SARS-CoV and MERS-CoV) and influenza virus ${ }^{6,7}$. Furthermore, using weather modeling, a recent study showed that it may be possible to predict the regions that are more likely to be at a higher risk of substantial community spread of COVID-19 in upcoming weeks ${ }^{8}$. Evidence-based research is needed to identify why the transmission of SARS-CoV-2 is higher in certain countries than in others.

\section{Existing comorbidities}

When predicting the impact of COVID19 on the African continent, however, it is important to recognize that the number of reported cases does not tell the whole story. Co-morbidity is of particular concern for Africa. $72 \%$ of COVID-19 cases admitted to the intensive care unit in Wuhan had a co-morbidity ${ }^{9}$ such as cardiovascular disease, diabetes or another condition. A recent study of sequentially hospitalized patients with confirmed COVID-19 in the New York City area showed that the most common co-morbidities at admission were hypertension $(56.6 \%)$, obesity $(41.7 \%)$ and diabetes $(33.8 \%)^{10}$. This might be a marker of social standing and as such could be an association rather than a causal link.

Although infectious disease still is a heavy burden on health in Africa - in 2017, AIDS caused $9.52 \%$ of all deaths in sub-Saharan Africa, followed by malaria at $7.27 \%$ and tuberculosis at $5.37 \%{ }^{11}$ — with a slowly aging population, urbanization and an increase in life expectancy ${ }^{12}$, Africa has seen a rise in non-communicable diseases ${ }^{13}$. Sub-Saharan Africa has seen an increase of $67 \%$ in overall non-communicable diseases in less than 20 years ${ }^{14}$. The prevalence of hypertension is estimated at $46 \%$ in adults over 25 years of age, and the number of adults with hypertension in 2025 is predicted to increase by about $60 \%$ to a total of 1.56 billion $^{15}$. In 2017, cardiovascular disease was responsible for $11.69 \%$ of all deaths in sub-Saharan Africa, which has only increased with time. This double burden of disease is particularly of concern for the management of COVID-19 on the continent.

Moreover, the strategy of 'flattening the curve, on which many countries of the Global North have relied, may not be relevant in Africa. Most African countries have over-burdened healthcare systems (i.e., not enough doctors and other medical providers, and not enough ventilators and dialysis machines, if patients develop severe forms of infection), and given the low socioeconomic status in most of African cities, mass quarantines may mean people will starve, as many of them already struggle to find food on a daily basis, much as has been reported in deprived neighborhoods in the USA.

Even if the response of caring for the patients with severe COVID-19 were to be fully managed by the weak health systems, that would mean those patients would taking precedence over many other patients who could be left in the lurch. It is estimated that about 769,000 people could die from malaria alone if funding and campaigns are suspended due to this COVID-19 pandemic ${ }^{16}$.

Luckily, thus far, the four countries that have been most heavily hit have the highest gross domestic products among African countries, and they also have the capacity to treat and monitor the spread of the disease more effectively than other African countries.

Because of those points, it is paramount that governments tailor their approaches to 
their own countries. A successful prevention method that will help to control the outbreak in Africa will need to be achieved by taking into consideration the social, economic and behavioral context of the African population.

Intensive and well-designed campaigns promoting adherence to public-health measures and active engagement in efforts to prevent spread are urgently needed. When the first cases were declared in African countries, it was reported that several patients fled the healthcare system to seek traditional or herbal medications because of a lack of trust in westernized medicine. The use of traditional medicine is a common practice that should be discouraged in this context.

Africa can also take a lesson from the USA, which attributes the rapid spread of the virus to misinformation and erroneous faulty beliefs and rhetoric that lowered people's perception of their risk ${ }^{17}$. Traditional and religious chiefs may have a major role to play in conjunction with local healthcare organizations and/or authorities.

Deploying well-trained mobile intervention groups that can provide in-home intervention (testing for SARS-CoV-2 and help in practicing the hygiene and safety policy) will help to prevent large crowds at hospitals, which overwhelm healthcare systems.

Africa is well equipped to control the situation if countries are able to apply fast and thorough contract tracing and monitoring, and ensure quarantine for potentially infectious contacts. Such an attitude has been shown to be the key in halting the Ebola outbreak very quickly in Nigeria ${ }^{18}$.

In conclusion, there is reason for hope in Africa's response to COVID-19. The pessimistic outlook and prediction of the pandemic in Africa can-and should-be prevented. To this end, African countries should keep implementing rapid action and remain vigilant in the upcoming weeks.

\section{Bamba Gaye (D) ${ }^{1,2 \bowtie}$, Stéphanie Khoury ${ }^{1,2}$, Crystal W. Cene $e^{1,3,4}$, Samuel Kingue 1,5 Roland N'Guetta ${ }^{1,6}$, Camille Lassale (iD) 7 ,} Dadhi Baldé1,8, Ibrahima Bara Diop ${ }^{1,9}$ Jennifer Beam Dowd ${ }^{10}$, Melinda C. Mills ${ }^{10}$ and Xavier Jouven 1,2,11

${ }^{1}$ The African Research Network, Kumasi, Ghana. ${ }^{2}$ Université de Paris, Integrative Epidemiology of Cardiovascular Diseases, Paris Cardiovascular Research Center-INSERM U970, Paris, France. ${ }^{3}$ Department of Medicine, Division of General Internal Medicine, University of North Carolina at Chapel Hill, Chapel Hill School of Medicine, Chapel Hill, NC, USA. ${ }^{4}$ Cecil G. Sheps Center for Health Services Research, University of North Carolina at Chapel Hill School of Medicine, Chapel Hill, NC, USA. ${ }^{5}$ University of Yaoundé, Ministry of Public Health, Yaoundé, Cameroon. ${ }^{6}$ Institute of Cardiology of Abidjan, Abidjan, Côte d'Ivoire. ${ }^{7}$ CIBER of Pathophysiology of Obesity and Nutrition, Instituto de Salud Carlos III, Madrid, Spain. ${ }^{8}$ Department of Cardiology, University Hospital of Conakry, Conakry, Guinea. ${ }^{9}$ Cardiology Department, University Hospital of Fann, Dakar, Senegal. ${ }^{10}$ Leverhulme Centre for
Demographic Science, Nuffield College, University of Oxford, Oxford, UK. ${ }^{11}$ Cardiology Department, Georges-Pompidou European Hospital, Paris, France.

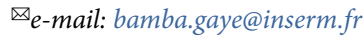

Published online: 11 June 2020

https://doi.org/10.1038/s41591-020-0960-y

References

1. Nkengasong, J. N. \& Mankoula, W. Lancet 395, 841-842 (2020).

2. Fanidi, A., Jouven, X. \& Gaye, B. Eur. Heart J. https://doi. org/10.1093/eurheartj/ehaa278 (2020).

3. Time https://time.com/5822461/coronavirus-tests-africa/ (2020).

4. Blavatnik School of Government \& University of Oxford. https://www.bsg.ox.ac.uk/research/research-projects/ coronavirus-government-response-tracker (accessed 26 April 2020).

5. Dowd, J.B. et al. Proc. Natl Acad. Sci. USA 117, 9696-9698 (2020)

6. Tan, J. et al. J. Epidemiol. Community Health 59, 186-192 (2005).

7. Chan, K. H. et al. Adv. Virol. 2011, 734690 (2011)

8. Sajadi, M.M. et al. Social Science Research Network https://doi. org/10.2139/ssrn.3550308 (2020).

9. Wang, D. et al. J. Am. Med. Assoc. 323, 1061-1069 (2020).

10. Richardson, S. et al. J. Am. Med. Assoc. 323, 2052-2059 (2020).

11. Global Health Data Exchange. http://ghdx.healthdata.org/gbdresults-tool (accessed 24 April 2020).

12. Foreman, K. J. et al. Lancet 392, 2052-2090 (2018).

13. Gaye, B. et al. BMJ Glob. Health 4, e001396 (2019).

14. Gouda, H. N. et al. Lancet Glob. Health 7, e1375-e1387 (2019).

15. Kearney, P. M. et al. Lancet 365, 217-223 (2005).

16. World Health Organization. https://www.who.int/publications-

detail/the-potential-impact-of-health-service-disruptionson-the-burden-of-malaria (2020).

17. Pew Research Center. https://www.people-press.org/2020/03/18/u-s -public-sees-multiple-threats-from-the-coronavirusand-concerns-are-growing/ (2020).

18. Fasina, F. O. et al. Euro Surveill. 19, 20920 (2014).

19. United Nations. https://population.un.org/wpp/ (2020).

20. Istituto Superiore di Sanità. https://www.epicentro.iss.it/

coronavirus/bollettino/Bollettino-sorveglianza-integrata-

COVID-19_30-marzo-2020.pdf (2020).

\title{
COVID-19 in Africa: the spread and response
}

\begin{abstract}
Given the current trends in incidence and underlying healthcare systems vulnerabilities, Africa could become the next epicenter of the COVID-19 pandemic. As the pandemic transitions to more widespread community transmission, how can the lessons learned thus far be consolidated to effectively curb the spread of COVID-19 while minimizing social disruption and negative humanitarian and economic consequences?
\end{abstract}

\section{Marguerite Massinga Loembé, Akhona Tshangela, Stephanie J. Salyer, Jay K. Varma, Ahmed E. Ogwell Ouma and John N. Nkengasong}

\footnotetext{
A s reports of COVID-19 emerged from Wuhan, China, in December 2019 (ref. $^{1}$ ), Africa started to prepare for the introduction of the first cases that would eventually arise from its close connections to China, a primary trade partner and host to more than 80,000 African students ${ }^{2}$. Statistical models based on air-travel data from China identified Egypt, Algeria and South Africa
}

as the countries at highest risk of initial introductions and spread in Africa ${ }^{3}$.

Drawing experience from the 2014 Ebola virus disease crisis in West Africa, African leaders were keenly aware that failure to contain COVID-19 would threaten health, prosperity and security ${ }^{4}$. African Union (AU) Member States rapidly focused on preventing COVID-19 importation and containing onward transmission within countries. As early as 2 January 2020, Ivory Coast $^{5,6}$, soon followed by other African countries, started implementing enhanced surveillance at airports, screening all passengers with a recent history of travel to China. In turn, most African airlines suspended direct flights to and from China? This approach initially seemed to pay off: the first COVID-19 case on the continent, with notification by Egypt on 14 February 